\title{
Advanced Energy Detector with Correlated Multiple Antennas
}

\author{
Sungtae $\mathrm{Kim}^{1}$ and Sungmook $\mathrm{Lim}^{2 *}$ \\ ${ }^{1}$ Communication System Examination Division, Korean Intellectual Property Office, Daejeon, Republic of Korea \\ [e-mail: always1977@naver.com] \\ 2 Department of IT-Energy Convergence (BK21 FOUR) and Department of Electronics Engineering, \\ Korea National University of Transportation, 50 Daehak-ro, Chungju-si, Chungbuk, 27469 Korea \\ [e-mail: smlim@ut.ac.kr] \\ ${ }^{*}$ Corresponding author: Sungmook Lim
}

Received August 24, 2021; revised October 15, 2021; accepted October 25, 2021; published December 31, 2021

\begin{abstract}
In cognitive radio networks where unlicensed secondary users opportunistically access to licensed spectrum unused by licensed primary users, spectrum sensing is one of the key issues in order to effectively use the frequency resource. For enhancing the sensing performance in energy detection-based spectrum sensing, spatial diversity based on multiple antennas is utilized. However, the sensing performance can be degraded when antennas are spatially correlated, resulting in inducing the harmful interference to primary users.

To overcome this problem, in this paper, an advanced energy detector is proposed. In the proposed sensing method, a weight matrix based on the eigenvalues of the spatial channels without any prior information on the primary signals is defined and utilized. In numerical simulations, it is shown that the proposed detector outperforms the conventional detector with regard to false-alarm and detection probabilities when antenna are spatially correlated.
\end{abstract}

Keywords: Energy detector, Antenna correlation, Sensing, Cognitive radio 


\section{Introduction}

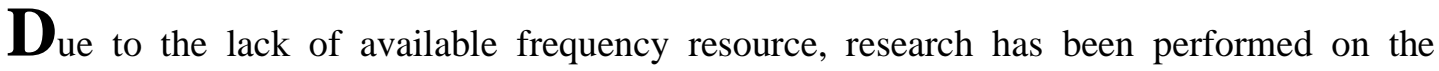
cognitive radio for efficient spectrum usage. In cognitive radio networks, opportunistic access to spectrum of licensed primary users is allowed to unlicensed secondary users under the condition that it does not cause catastrophic interference to primary users [1]. To prevent interfering communications among primary users, in advance of accessing to licensed spectrum, secondary users should identify the available spectrum for secondary users with spectrum sensing. Spectrum sensing employing various detection techniques finds the portions of spectrum currently unused by primary users. Spectrum sensing is therefore one of key issues for realization of cognitive radio networks.

Recently, lots of spectrum sensing methods have been studied. Those of sensing techniques can be categorized into two groups. One of the groups assumes that the secondary users have prior knowledge of the primary user signals (e.g., channel parameters, modulation scheme, and statistical features of the primary user signals). Sensing technique based on the matched filter, which requires channel knowledge, is introduced in [2]. There are also feature detection techniques that utilize statistical features (e.g., covariance, cyclostationary) of the primary user signals [3-5]. The other group of sensing techniques assumes mostly no prior information and is based on the simple received energy measurement [6-13]. Although the first group outperforms the second group using energy detection technique in terms of the sensing performance, the first group has more computational complexity and less generality. Generally, in cognitive radio networks, heterogeneous wireless communication systems which use each licensed spectrum may coexist in the same area. In this case, the first group that requires prior information is too expensive to detect multiple licensed spectra. Accordingly, the second group using energy detection is suitable in heterogeneous wireless communication systems. In this reason, spectrum sensing based on energy detection has been studied in depth [14].

In cognitive radio networks, secondary users should be located far away from primary users, because it does not cause the harmful interference to primary users. In this circumstance, the signal to noise ratio (SNR) of the signal transmitted by the primary user is mostly less than $0 \mathrm{~dB}$ at the secondary users, which degrades the sensing performance of energy detectors because of the lack of the detection sensitivity [15] [16]. If a secondary user fails to detect received primary user signals, then secondary user signal causes fatal interference to the primary receivers. Adversely, if secondary user makes false alarm in spectrum sensing, then transmission opportunity is missed and it is concluded to throughput degradation of the secondary system. Therefore, sensing performance enhancement of energy detection-based sensing techniques should be required.

At a single sensing node of a secondary system, research for performance enhancement of energy detection-based sensing has focused on utilizing spatial diversity by employing multiple antennas [7-11]. These researches show that employing multiple antennas is quite effective for sensing performance enhancement. However, considering antenna correlation for feasibility, the achievable spatial diversity gain is rather deteriorated [10][11]. Hence, the demand for an advanced sensing technique which can overcome the problem due to spatially correlated antennas is come to the fore. To cope with adverse effect of correlation, eigenvalue-based methods can be good solutions. Maximum eigenvalue detection [17], GLRT based signal-subspace eigenvalues method [18], maximum-minimum eigenvalues detection 
[19] and optimal eigenvalue weighting detection [20] are representative eigenvalue-based sensing schemes. These can achieve much better sensing performance than energy detection with antenna correlation. However, these eigenvalue-based methods also require information of eigenvalues. Moreover, since these methods deal with eigenvalues, much higher computational complexity than energy detection-based sensing is inevitable. It gives us a motivation to propose a new sensing scheme which shows better sensing performance than conventional energy detection-based sensing techniques even with antenna correlation and has reasonable computation complexity.

In order to overcome the poor sensing performance caused by the correlation between antennas, in this paper, the enhanced energy detector for sensing by the secondary user with multiple antennas is proposed. Although the proposed sensing technique does not require more information on primary signals than the conventional energy detector, it outperforms the conventional energy detector in terms of the sensing performance under the condition where spatial correlation between antennas exists. Also, since the proposed sensing technique does not need to deal with channel eigenvalues, it has much less computational complexity than that of eigenvalue-based methods. The detection and false-alarm probabilities of the proposed detector are derived. Also, the performance is analyzed and based on that, it is verified that the proposed detector is superior to the conventional method.

The remainder of this paper is organized as follows: Section 2 introduces the system model of spectrum sensing by energy detector with multiple antennas. Then, Section 3 proposes the advanced detector and analyzes the sensing performance of the proposed method. In Section 4, numerical results are shown, and finally Section 5 shows concluding remarks of this paper.

\section{Sensing Performance with Multi-Antenna}

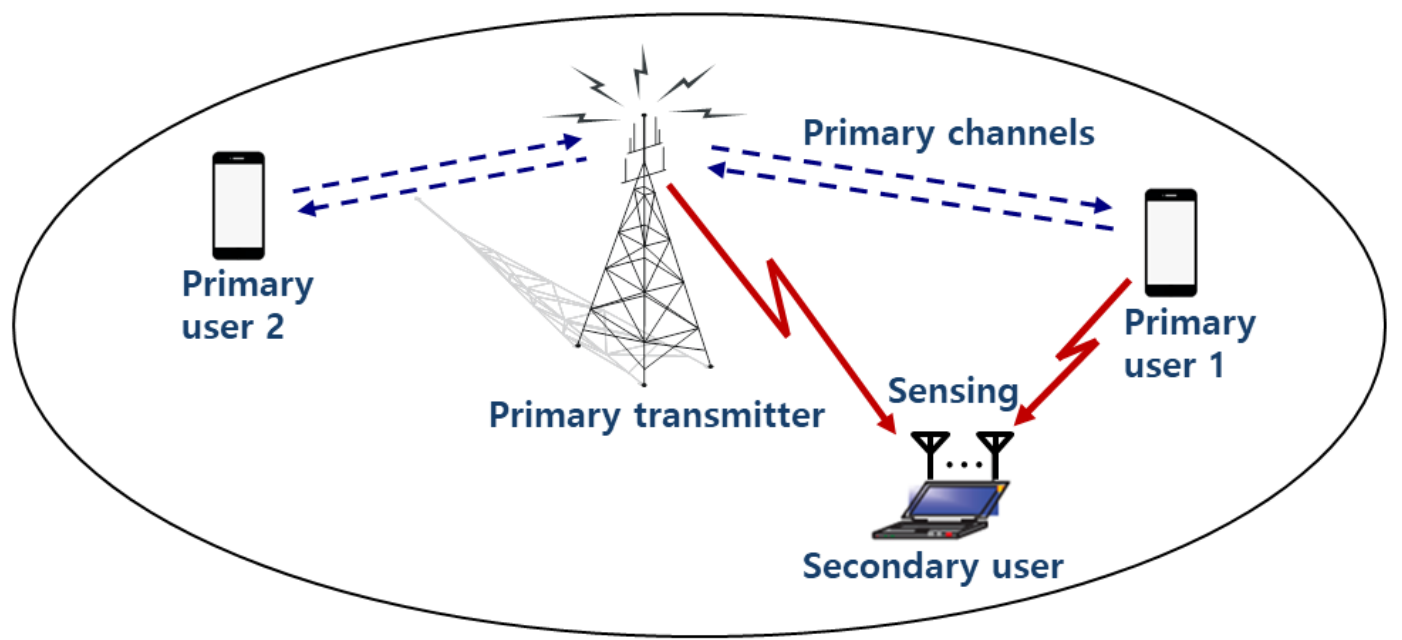

Fig. 1. System model of spectrum sensing by energy detector with multiple antennas

\subsection{System Model of Spectrum Sensing with Multi-Antenna}

In this paper, as shown in Fig. 1, a secondary user equipped with $M$ antennas performs spectrum sensing. For this, the binary hypothesis test is performed as follows: 


$$
\begin{aligned}
& H_{0}: x_{i}(k)=n_{i}(k), i=1,2, \ldots, M \\
& H_{1}: x_{i}(k)=h_{i}(k) s(k)+n_{i}(k), i=1,2, \ldots, M,
\end{aligned}
$$

where $x_{i}(k)$ is the received signal by the secondary transmitter at the $i$ th antenna of the $k$ th time instant. $n_{i}(k)$ means the additive white Gaussian noise with zero mean and variance $\sigma_{n}^{2}$, i.e., $n_{i}(k): \mathbf{C N}\left(0, \sigma_{n}^{2}\right)$, and it is assumed to be independent and identically distributed (i.i.d.). $h_{i}(k)$ denotes the complex channel coefficient following Rayleigh distribution, i.e., $h_{i}(k): \mathbf{C N}\left(0, \sigma_{h}^{2}\right)$. Finally, $s(k)$ is the signal that the primary user transmits with M-ary phase shift keying (MPSK) modulation. It is also assumed that the received signal power is $P$ when the secondary user receives $s(k)$. Since we aim to analyze how spatial correlation affects sensing performance, the channel coefficients $h_{i}(k)$ are assumed to be correlated between antennas, whereas independent in the time domain. In addition, it is assumed that $s(k), h_{i}(k)$ and $n_{i}(k)$ are independent of each other.

In general, the spatial correlation is modelled as the exponential correlation because of its simplicity and accuracy [21], so the exponential correlation model is also considered as spatial correlation in this paper. When the number of the antennas is $M$, the matrix of the antenna correlation, $R$, can be defined as follows:

$R$ which denotes the antenna correlation matrix is defined as

$$
R_{i j}=\left\{\begin{array}{ll}
\rho^{i-j}, & i \leq j \\
R_{j i}^{*}, & i>j
\end{array}, i, j=1, \mathrm{~L}, M, \quad 0 \leq \rho \leq 1\right.
$$

where the size of $R$ is $M \times M$ and $\rho$ is the correlation coefficient between two adjacent antennas. It is noted that the correlation coefficients have only real values, because the real and imaginary parts of $h_{i}(k)$ are independent of each other. Therefore, the correlation coefficient, $\rho$, can be approximated as defined in [22]

$$
\rho=e^{-23 \Lambda^{2}\left(\frac{d}{\lambda_{c}}\right)^{2}},
$$

where $\lambda_{c}, \Lambda$ and $d$ denote the wavelength of the signal, the angular spread and the antenna spacing between two contiguous antennas, respectively. As a result, $R$ becomes a symmetric Toeplitz matrix.

\subsection{Energy Detector with Multiple Antennas}

Sensing performance of the secondary user with multi-antenna is investigated in [11]. The energy of the received signal is sensed during the sensing period by the energy detector, and then, whether the primary user uses the spectrum or not is determined by comparing the estimated energy with a predefined threshold. When the sensing period is composed of $N$ 
samples in the time domain, the test statistic for energy detection can be

$$
T_{e}(\bar{X})=\bar{X}^{H} \bar{X}=\sum_{k=1}^{N} \sum_{i=1}^{M}\left|X_{i}(k)\right|^{2}
$$

The distribution of the test statistics $T_{e}(\bar{X})$ can be expressed as

$$
\begin{array}{ll}
H_{0}: & T_{e}(\bar{X}): \mathbf{N}\left(N M \sigma_{n}^{2}, N M \sigma_{n}^{4}\right) \\
H_{1}: & T_{e}(\bar{X}): \mathbf{N}\left(N M\left(P \sigma_{h}^{2}+\sigma_{n}^{2}\right), N \sum_{i=1}^{M}\left(P \sigma_{h}^{2} \lambda_{i}+\sigma_{n}^{2}\right)^{2}\right) .
\end{array}
$$

With decision threshold $\tau_{e}$, the detection and false-alarm probabilities can be

$$
P_{D e}=Q\left[\frac{\tau_{e}-N M\left(P \sigma_{h}^{2}+\sigma_{n}^{2}\right)}{\sqrt{N \sum_{i=1}^{M}\left(P \sigma_{h}^{2} \lambda_{i}+\sigma_{n}^{2}\right)^{2}}}\right]
$$

and

$$
P_{F e}=Q\left[Q^{-1}(\alpha) \sqrt{\frac{1}{M} \sum_{i=1}^{M}\left(\gamma \lambda_{i}+1\right)^{2}}+\gamma \sqrt{N M}\right],
$$

respectively. $Q(x)=\frac{1}{\sqrt{2 \pi}} \int_{x}^{\infty} e^{-t^{2} / 2} d t$ means the tail probability of the Gaussian probability density function (PDF) with zero mean and unit variance, and $\gamma=P \sigma_{h}^{2} / \sigma_{n}^{2}$ means the received SNR at the secondary user.

When the target detection probability should satisfy the following conditions, $P_{D e} \geq \alpha$, the decision threshold, $\tau_{e}$, can be derived from (6) as

$$
\tau_{e}=Q^{-1}(\alpha) \sqrt{N \sum_{i=1}^{M}\left(P \sigma_{h}^{2} \lambda_{i}+\sigma_{n}^{2}\right)^{2}}+N M\left(P \sigma_{h}^{2}+\sigma_{n}^{2}\right) .
$$

\subsection{Spatially Correlated Antennas}

As for the energy detector with multi-antenna, the false-alarm and detection probabilities are optimum for independent antennas [14]. When antennas become correlated, however, the sensing performance is worse than that of the independent antenna case. Based on (6) and (7), the sensing performance of the energy detector with multi-antenna can be investigated. Regardless of values of $M, N$ and $\gamma$, it is obvious that 


$$
\begin{aligned}
& Q^{-1}\left(\lim _{\rho \rightarrow 0} P_{F e}\right)-Q^{-1}\left(P_{F e}\right) \geq 0, \\
& Q^{-1}\left(\lim _{\rho \rightarrow 0} P_{D e}\right)-Q^{-1}\left(P_{D e}\right) \leq 0 .
\end{aligned}
$$

Therefore, regardless of $M$, the false-alarm probability monotonically increases and the detection probabilty monotonically decreases according to the antenna correlation coefficient, respectively. As a result, the following relationship can be determined:

$$
\begin{aligned}
& \lim _{\rho \rightarrow 0} P_{F e} \leq \lim _{\rho \rightarrow 1} P_{F e}, \\
& \lim _{\rho \rightarrow 0} P_{D e} \geq \lim _{\rho \rightarrow 1} P_{D e} .
\end{aligned}
$$

When the antenna correlation occurs, the performance of the energy detector deteriorates. Especially, the higher the spatial correlation between antennas is, the worse the sensing performance is [11].

\section{Advanced Energy Detector}

\subsection{Proposed Detector}

In the previous chapter, we have investigated how the spatial correlation between antennsa affects the performance of the energy detector. Even if the energy detector shows optimum performance with i.i.d. received signals, its sensing performance is degraded with correlated antennas. Hence, an advanced detector which can overcome the adverse effect of spatial correlation is required.

With spatially correlated channels, to achieve better sensing performance, the energy of primary samples received at each antenna should be adequately weighted. However, in general, a secondary sensing node does not know any information on the primary signals including the eigenvalues of spatial channels. Therefore, it is required to find the weight matrix for the received signals, in which the weights are in proportion to the eigenvalues of the spatial channels and can be determined without any prior information on the primary signals.

Let us define the proposed detector with the test statistic $T_{p}(\bar{X})$ as follows [23]:

$$
T_{p}(\bar{X})=\bar{X}^{H} W_{p} \bar{X} \underset{H_{0}}{>} \tau_{p}^{H_{1}},
$$

where $\tau_{p}$ is the threshold for the proposed detector and $W_{p}$ is the weight matrix for the proposed detector. Since the received signals are independent for time index $k$, the test statistic $T_{p}(\bar{X})$ can be expressed with the partial statistic $T_{p}(\bar{X}(k))$ as follows: 


$$
\begin{aligned}
& T_{p}(\bar{X})=\sum_{k=1}^{N} T_{p}(\bar{X}(k)) \\
& T_{p}(\bar{X}(k))=\bar{X}^{H}(k) W_{p k} \bar{X}(k),
\end{aligned}
$$

where $W_{p k}$ is a $M \times M$ diagonal block of a block diagonal matrix $W_{p}$. If the probability of which there is primary signals is $v$, then $H_{1}$ and $H_{0}$ occur with the probabilities $v$ and $1-v$, respectively. Then, the $M \times M$ covariance matrix of the received signal vector $X(k)$ can be

$$
\begin{aligned}
\Sigma_{\bar{X}(k)} & =E\left[\bar{X}(k) \bar{X}^{H}(k)\right] \\
& =\lim _{N \rightarrow \infty} \frac{1}{N} \sum_{k=1}^{N} \bar{X}(k) \bar{X}^{H}(k) \\
& =\sum_{i=1}^{M}\left(v P \sigma_{h}^{2} \lambda_{i}+\sigma_{n}^{2}\right) u_{i} u_{i}^{H} .
\end{aligned}
$$

Without any prior knowledge of the transmitted signal by the primary suser, $\Sigma_{\bar{X}(k)}$ can be easily approximated as follows:

$$
\hat{\Sigma}_{\bar{X}(k)}=\frac{1}{N} \sum_{k=1}^{N} \bar{X}(k) \bar{X}^{H}(k)
$$

Moreover, for sufficiently large $N$, the difference between $\Sigma_{\bar{X}(k)}$ and $\hat{\Sigma}_{\bar{X}(k)}$ is almost disappeared. Hence, $\Sigma_{\bar{X}(k)}$ can be obtained blindly at a secondary sensing node. Though the extent of reflecting the channel gains of spatial channels varies with the value of $v$, the eigenvalues of $\Sigma_{\bar{X}(k)}$ is in proportion to the eigenvalues of $R$. Therefore, $\Sigma_{\bar{X}(k)}$ is adequate for the weight matrix, because its eigenvalues are in proportion to the eigenvalues of $R$, and moreover, it can be obtained totaly blindly. So the $M \times M$ diagonal block of the proposed weight matrix can be defined as

$$
W_{p k}=\sum_{i=1}^{M}\left(v P \sigma_{h}^{2} \lambda_{i}+\sigma_{n}^{2}\right) u_{i} u_{i}^{H}
$$

Then, the partial statistic of the proposed detector can be

$$
\begin{aligned}
T_{p}(\bar{X}(k)) & =\bar{X}^{H}(k)\left(\sum_{i=1}^{M}\left(v P \sigma_{h}^{2} \lambda_{i}+\sigma_{n}^{2}\right) u_{i} u_{i}^{H}\right) \bar{X}(k) \\
& =\sum_{k=1}^{N} Y_{p i}^{H}(k) Y_{p i}(k) \\
Y_{p i}(k) \quad & =\left[v P \sigma_{h}^{2} \lambda_{i}+\sigma_{n}^{2}\right]^{1 / 2} u_{i}^{H} \bar{X}(k) .
\end{aligned}
$$

Finally, the proposed blindly combined detector is defined as 


$$
\begin{aligned}
T_{p}(\bar{X}) & =\sum_{k=1}^{N} T_{p}(\bar{X}(k)) \\
& =\sum_{k=1}^{N} Y_{p i}^{H}(k) Y_{p i}(k)_{\substack{H_{0} \\
<}}^{>} \tau_{p} .
\end{aligned}
$$

\subsection{Performance Analysis of Proposed Detector}

In (16), since $\left\{u_{i}\right\}_{i=1}^{M}$ are orthonormal eigenvectors of $W_{p k},\left\{Y_{p} i(k)\right\}_{i=1}^{M}$ are independent complex Gaussian random variables which are distributed as follows [24]:

$$
\begin{aligned}
& H_{0}: Y_{p i}(k): \mathbf{C N}\left(0, v P \sigma_{h}^{2} \sigma_{n}^{2} \lambda_{i}+\sigma_{n}^{4}\right) \\
& H_{1}: Y_{p i}(k): \mathbf{C N}\left(0, v P^{2} \sigma_{h}^{4} \lambda_{i}^{2}+(1+v) P \sigma_{h}^{2} \sigma_{n}^{2} \lambda_{i}+\sigma_{n}^{4}\right) .
\end{aligned}
$$

Since $Y_{p i}(k)$ is a complex Gaussian random variable, $Y_{p i}^{H}(k) Y_{p i}(k)$ becomes a gamma random variable. Then, under $H_{0}$ and $H_{1}$, the PDF of $Y_{p i}^{H}(k) Y_{p i}(k)$ is given by

$$
p\left(Y_{p i}^{H}(k) Y_{p i}(k) \mid H_{0}\right)=\frac{e^{-\chi /\left(v P \sigma_{h}^{2} \sigma_{n}^{2} \lambda_{i}+\sigma_{n}^{4}\right)}}{v P \sigma_{h}^{2} \sigma_{n}^{2} \lambda_{i}+\sigma_{n}^{4}}
$$

and

$$
p\left(Y_{p i}^{H}(k) Y_{p i}(k) \mid H_{1}\right)=\frac{e^{-x /\left(v P^{2} \sigma_{h}^{4} \lambda_{i}^{2}+(1+v) P \sigma_{h}^{2} \sigma_{n}^{2} \lambda_{i}+\sigma_{n}^{4}\right)}}{v P^{2} \sigma_{h}^{4} \lambda_{i}^{2}+(1+v) P \sigma_{h}^{2} \sigma_{n}^{2} \lambda_{i}+\sigma_{n}^{4}},
$$

respectively [25]. The partial statistic $T_{p}(\bar{X}(k))$ is a gamma random variable, and since $\left\{T_{p}(\bar{X}(k))\right\}_{k=1}^{N}$ are independent for $k$, the test statistic of the proposed detector $T_{p}(\bar{X})$ can be obtained by the central limit theorem (CLT). Then, the test statistic $T_{p}(\bar{X})$ under $H_{0}$ and $H_{1}$ are asymptotically normally distributed as follows:

$$
\begin{aligned}
H_{0}: T_{p}(\bar{X}): \mathbf{N}( & N \sum_{i=1}^{M} v P \sigma_{h}^{2} \sigma_{n}^{2} \lambda_{i}+\sigma_{n}^{4}, \\
& \left.N \sum_{i=1}^{M}\left(v P \sigma_{h}^{2} \sigma_{n}^{2} \lambda_{i}+\sigma_{n}^{4}\right)^{2}\right) .
\end{aligned}
$$

and 


$$
\begin{aligned}
H_{1}: T_{p}(\bar{X}): \mathbf{N}( & N \sum_{i=1}^{M} v P^{2} \sigma_{h}^{4} \lambda_{i}^{2}+(1+v) P \sigma_{h}^{2} \sigma_{n}^{2} \lambda_{i}+\sigma_{n}^{4}, \\
& \left.N \sum_{i=1}^{M}\left(v P^{2} \sigma_{h}^{4} \lambda_{i}^{2}+(1+v) P \sigma_{h}^{2} \sigma_{n}^{2} \lambda_{i}+\sigma_{n}^{4}\right)^{2}\right),
\end{aligned}
$$

respectively. With the decision rule in (11), the detection and false-alarm probabilities are derived with the PDFs of the test statistic $T_{p}(\bar{X})$ under $H_{0}$ and $H_{1}$ as follows [23]:

$$
P_{D p}=\operatorname{Pr}\left\{T_{p}(\bar{X})>\tau_{p} ; H_{1}\right\}=Q\left[\frac{\tau_{p}-N \sum_{i=1}^{M} v P^{2} \sigma_{h}^{4} \lambda_{i}^{2}+(1+v) P \sigma_{h}^{2} \sigma_{n}^{2} \lambda_{i}+\sigma_{n}^{4}}{\sqrt{N \sum_{i=1}^{M}\left(v P^{2} \sigma_{h}^{4} \lambda_{i}^{2}+(1+v) P \sigma_{h}^{2} \sigma_{n}^{2} \lambda_{i}+\sigma_{n}^{4}\right)^{2}}}\right]
$$

and

$$
P_{F p}=\operatorname{Pr}\left\{T_{p}(\bar{X})>\tau_{p} ; H_{0}\right\}=Q\left[\frac{\tau_{p}-N \sum_{i=1}^{M} v P \sigma_{h}^{2} \sigma_{n}^{2} \lambda_{i}+\sigma_{n}^{4}}{\sqrt{N \sum_{i=1}^{M}\left(v P \sigma_{h}^{2} \sigma_{n}^{2} \lambda_{i}+\sigma_{n}^{4}\right)^{2}}}\right],
$$

respectively. With a target detection probability constraint $P_{D p} \geq \alpha$, from (23), $\tau_{p}$ is determined to minimize $P_{F p}$ as follows:

$$
\begin{array}{r}
\tau_{p}=Q^{-1}(\alpha) \sqrt{N \sum_{i=1}^{M}\left(v P^{2} \sigma_{h}^{4} \lambda_{i}^{2}+(1+v) P \sigma_{h}^{2} \sigma_{n}^{2} \lambda_{i}+\sigma_{n}^{4}\right)^{2}} \\
+N \sum_{i=1}^{M} v P^{2} \sigma_{h}^{4} \lambda_{i}^{2}+(1+v) P \sigma_{h}^{2} \sigma_{n}^{2} \lambda_{i}+\sigma_{n}^{4}
\end{array}
$$

Then, with $P_{D p} \geq \alpha, P_{F p}$ of the proposed blindly combined detector can be calculated as

$$
P_{F p}=Q\left[\frac{Q^{-1}(\alpha) \sqrt{\sum_{i=1}^{M}\left(v \gamma^{2} \lambda_{i}^{2}+(1+v) \gamma \lambda_{i}+1\right)^{2}}+\sqrt{N} \sum_{i=1}^{M} v \gamma^{2} \lambda_{i}^{2}+\gamma \lambda_{i}}{\sqrt{\sum_{i=1}^{M}\left(v \gamma \lambda_{i}+1\right)^{2}}}\right] .
$$

On the other hand, with a target false-alarm probability constraint $P_{F p} \leq \beta$, the decision threshold $\tau_{p}$ should be determined to maximize $P_{D p}$ as follows: 


$$
\tau_{p}=Q^{-1}(\beta) \sqrt{N \sum_{i=1}^{M}\left(v P \sigma_{h}^{2} \sigma_{n}^{2} \lambda_{i}+\sigma_{n}^{4}\right)^{2}}+N \sum_{i=1}^{M} v P \sigma_{h}^{2} \sigma_{n}^{2} \lambda_{i}+\sigma_{n}^{4} .
$$

Then, with $P_{F p} \leq \beta, P_{D p}$ is given by

$$
P_{D p}=Q\left[\frac{Q^{-1}(\beta) \sqrt{\sum_{i=1}^{M}\left(v \gamma \lambda_{i}+(1-v)\right)^{2}}-\sqrt{N}\left(\sum_{i=1}^{M} v \gamma^{2} \lambda_{i}^{2}+(1-v) \gamma \lambda_{i}\right)}{\sqrt{\sum_{i=1}^{M}\left(v \gamma^{2} \lambda_{i}^{2}+\gamma \lambda_{i}+(1-v)\right)^{2}}}\right] .
$$

\subsection{Performance Comparison with Conventional Energy Detector}

By the derived detection and false alarm probabilities above, we compare the proposed detector with the conventional energy detector in terms of the performance of the false-alarm and detection probabilities. The sensing performance comparison is performed when $\rho=0$ (antennas are uncorrelated) and $\rho=1$ (antennas are fully correlated).

With the antenna correlation matrix $R$, when antennas are uncorrelated $(\rho=0)$, all the eigenvalues of $R$ are equal to 1 . Then, the detection and false-alarm probabilities are the same as those of the energy detector, respectively,

$$
\lim _{\rho \rightarrow 0} P_{D p}=\lim _{\rho \rightarrow 0} P_{D e}, \lim _{\rho \rightarrow 1} P_{D p}=\lim _{\rho \rightarrow 1} P_{D e}
$$

On the other hand, when $\rho=1$, the eigenvalues become zero except the maximum eigenvalue. Then, $P_{D p}$ and $P_{F p}$ are calculated as follows:

$$
\lim _{\rho \rightarrow 1} P_{D p}=Q\left[\frac{Q^{-1}(\beta) \sqrt{1+\frac{(1-v)^{2}(M-1)}{(v \gamma M+(1-v))^{2}}}-\gamma M \sqrt{N}}{\sqrt{(\gamma M+1)^{2}+\frac{(1-v)^{2}(M-1)}{(v \gamma M+(1-v))^{2}}}}\right],
$$




$$
\lim _{\rho \rightarrow 1} P_{F p}=Q\left[\frac{Q^{-1}(\alpha) \sqrt{(\gamma M+1)^{2}+\frac{(M-1)}{(v \gamma M+1)^{2}}}+\gamma M \sqrt{N}}{\sqrt{1+\frac{(M-1)}{(v \gamma M+1)^{2}}}}\right] .
$$

$P_{D p}$ and $P_{F p}$ of the proposed detector vary with the probability of the presence of primary signals, $v$. In (30), when $v$ is equal to 0 and 1 , the values of the term in the Q-function are

$$
\lim _{\rho \rightarrow 1} Q^{-1}\left(P_{D p}\right)=\frac{Q^{-1}(\beta)-\gamma M \sqrt{N}}{(\gamma M+1)},(v=0),
$$

and

$$
\lim _{\rho \rightarrow 1} Q^{-1}\left(P_{D p}\right)=\frac{Q^{-1}(\beta)-\gamma \sqrt{N M}}{\sqrt{\gamma^{2} M+2 \gamma+1}},(v=1),
$$

respectively. In (30), $\frac{(1-v)^{2}(M-1)}{(v \gamma M+(1-v))^{2}}$ is monotonically decreasing function of $v$. Hence, the upper and the lower bounds of the asymptotic detection probability of the proposed detector are the same as that of the energy detector,

$$
\lim _{\rho \rightarrow 1} P_{D p} \geq \lim _{\rho \rightarrow 1} P_{D e}
$$

On the other hand, in case of the asymptotic false-alarm probability in (31), when $v$ becomes 0 and 1 , the values of the term in the Q-function are calculated as

$$
\lim _{\rho \rightarrow 1} Q^{-1}\left(P_{F p}\right)=Q^{-1}(\alpha) \sqrt{\gamma^{2} M+2 \gamma+1}+\gamma \sqrt{N M},(v=0),
$$

and

$$
\lim _{\rho \rightarrow 1} Q^{-1}\left(P_{F p}\right)=Q^{-1}(\alpha) \sqrt{\frac{\left(\gamma^{4} M^{3}+4 \gamma^{3} M^{2}+6 \gamma^{2} M+4 \gamma+1\right)}{\left(\gamma^{2} M+2 \gamma+1\right)}}+\frac{\gamma \sqrt{N M}(\gamma M+1)}{\sqrt{\gamma^{2} M+2 \gamma+1}},(v=1)
$$

In (36), for reasonable performance in the false-alarm probability, $P_{F e}$ and $P_{F p} \leq 0.5$. Then, for any values of $M, N$ and $\gamma$, it is obvious that 


$$
\lim _{\rho \rightarrow 1, \nu=1} Q^{-1}\left(P_{F p}\right) \geq \lim _{\rho \rightarrow 1} Q^{-1}\left(P_{F e}\right) .
$$

Hence, since the $Q(x)$ is monotonic decreasing function of $x$, the asymptotic false alarm probability of the conventional method is the upper bound of that of the proposed detector. Therefore, regardless of antenna correlation, the proposed detector constantly outperforms the conventional method for all $v$ values.

\section{Numerical Results}

In this section, we compare the proposed detector with the conventional detector with regard to the sensing performance through numerical simulations. Fig. $2\left(d=\lambda_{c} / 8, \Lambda=0.5^{\circ}\right)$ compares the proposed detector with the conventional scheme in terms of the false-alarm probability according to antenna correlation. The simulation result is well matched with our sensing perfromance analysis. As we already verified with numerical analysis in the previous chapter, the proposed detector is better than the conventional scheme. Specifically, as the spatial correlation bwteen antennas is higher, the false-alarm probability of the proposed detector gets worse. However, the performance is still superior to that of the conventional technique. In this reason, the performance of the conventional method with 6 antennas is crossing with that of the proposed detector with 4 antennas. In Fig. $3\left(d=\lambda_{c} / 2\right)$, the false-alarm probability of the proposed method is shown according to values of angular spread when SNR is $0 d B$. As we analyzed in the previous chapter, the false-alarm probability of the proposed scheme is degraded as antenna correlation increases. Since the weight matrix of the proposed detector is in proportion to eigenvalue of each spatial channel, the sensing performance is better with correlated antennas than that with independent antennas. In Table 1, false-alarm probability of the proposed detector is compared with that of the conventional energy detector with various extent of antenna correlation ( $\mathrm{SNR}=0 d B, 4 \mathrm{Rx}$ antennas). When all of $\mathrm{Rx}$ antennas are independent, the sensing performance of the proposed detector is the same as that of the conventional energy detector. However, the sensing performance of the conventional energy detector is deteriorated as antenna correlation increases. In contrast to the conventional energy detector's case, the sensing performance of the proposed detector is improved with antenna correlation, because the proposed detector adequately combines the received energy from each Rx antenna with the weight matrix. Hence, the proposed detector achieves much better sensing performance comparing to energy detector with antenna correlation.

The weight matrix of the proposed method is obtained by averaging the covariance matrix. Hence, it requires some number of samples until the weight matrix have converged. Fig. 4 $\left(d=\lambda_{c} / 2, \Lambda=0.5^{\circ}\right)$ illustrates mean squared error between the ideal weight matrix and the weight matrix obtained by averaging for 10000 time samples. From the figure, it can be concluded that over 1000 time samples are required for convergence of the weight matrix. To verify the extent of the convergence, in Fig. $5\left(d=\lambda_{c} / 2, \Lambda=0.5^{\circ}\right)$, we illustrate the false-alarm probabilities of the proposed scheme for $M=4$ with and without averaging time for 1000 time samples. Based on Fig. 5, it is confirmed that the proposed detector requires at 
least order of 1000 time samples for averaging time. It is a weak point of the proposed method compared to the energy detector. However, if the secondary system in CR networks not consider the mobility like IoT systems, the averaging time for the proposed detector can be allowed.

Furthermore, computational complexity of the proposed detector is a little bit higher than that of the energy detector, but it is much lower than that of the eigenvalue-based method. The computational complexity of the energy detector is $O(M N)$, where $M$ is the number of received antenna and $N$ is the number of received samples [21], and the computational complexity of the eigenvalue-based sensing is $K^{2} L N M+O\left(K^{2} M^{3} L^{3}\right)$, where $K$ is the oversampling factor and $L$ is the smoothing factor [19]. Since the proposed detector requires averaging process for weight matrix, the computational complexity of the proposed detector is $T M N+O(2 M N)$, where $T$ is the number of averaging samples. It means the proposed detector shows better sensing performance and has reasonable computation complexity.

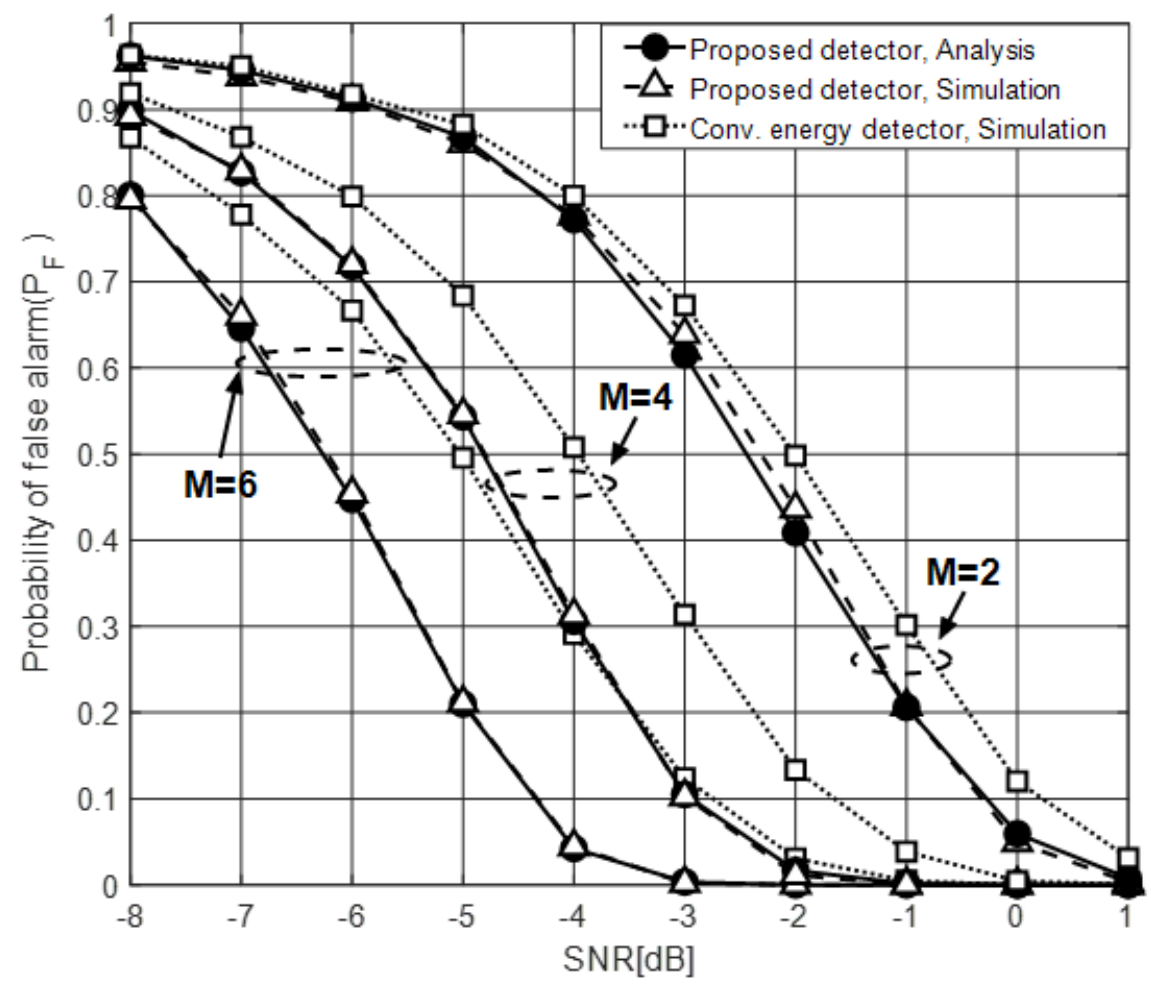

Fig. 2. False alarm probabilities of the proposed and conventional detectors as the number of antennas 


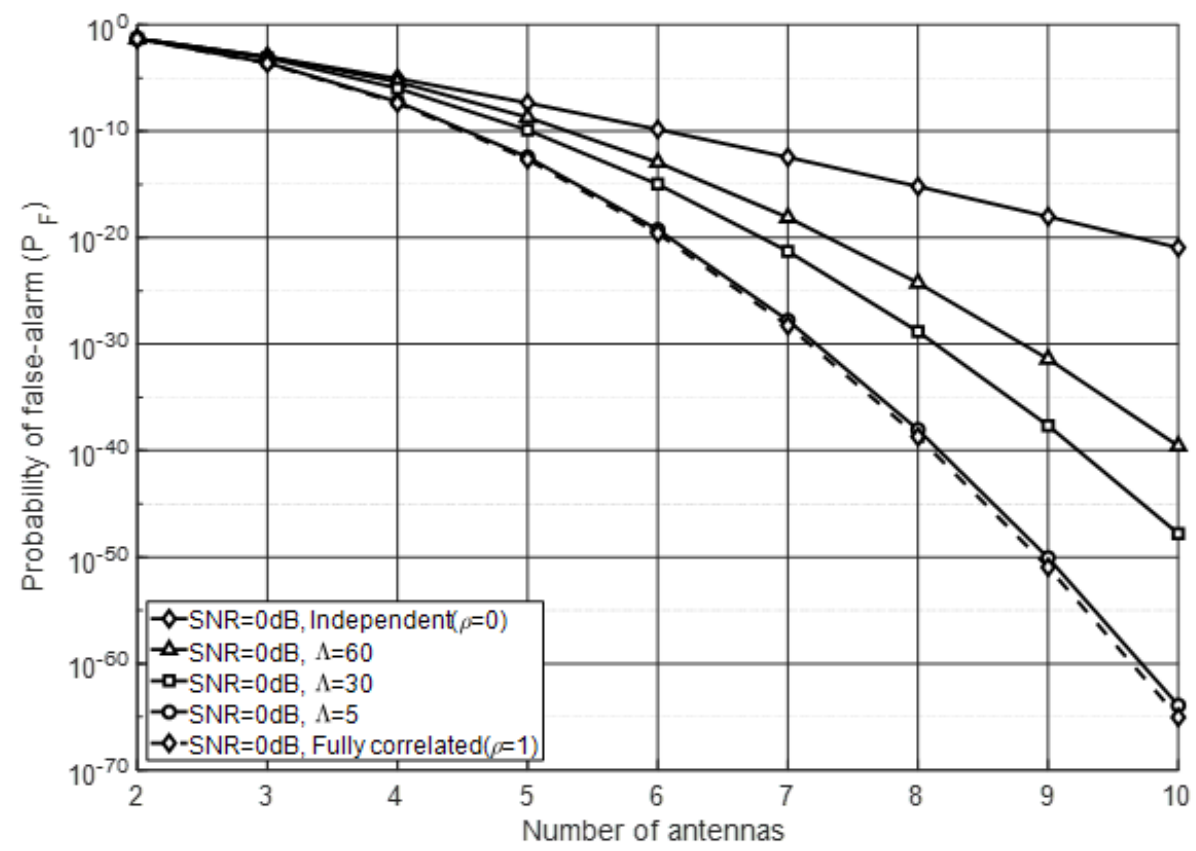

Fig. 3. False alarm probabilities of the proposed detector according to values of the angular spread.

Table. 1. False alarm probabilities of the conventional and proposed detectors with various antenna correlation

\begin{tabular}{|c|c|c|c|c|c|}
\hline Ant. Corr. & Independent & $\Lambda=60$ & $\Lambda=30$ & $\Lambda=5$ & Fully corr. \\
\hline Conv. ED & $8.871 \times 10^{-6}$ & $2.257 \times 10^{-5}$ & $1.742 \times 10^{-4}$ & 0.00232 & 0.00264 \\
\hline Proposed & $8.870 \times 10^{-6}$ & $4.175 \times 10^{-6}$ & $1.084 \times 10^{-6}$ & $5.799 \times 10^{-8}$ & $4.359 \times 10^{-8}$ \\
\hline
\end{tabular}

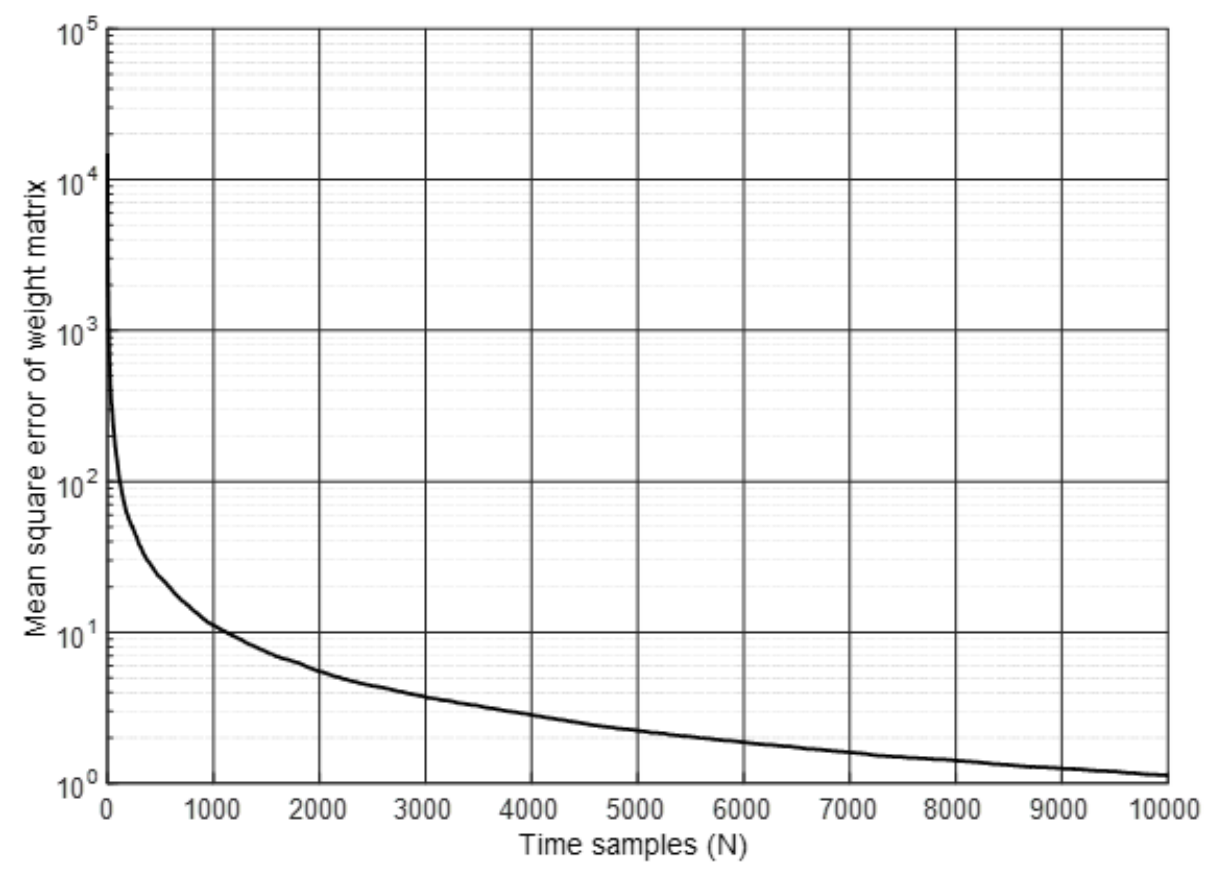

Fig. 4. Mean square error of the weight matrix. 


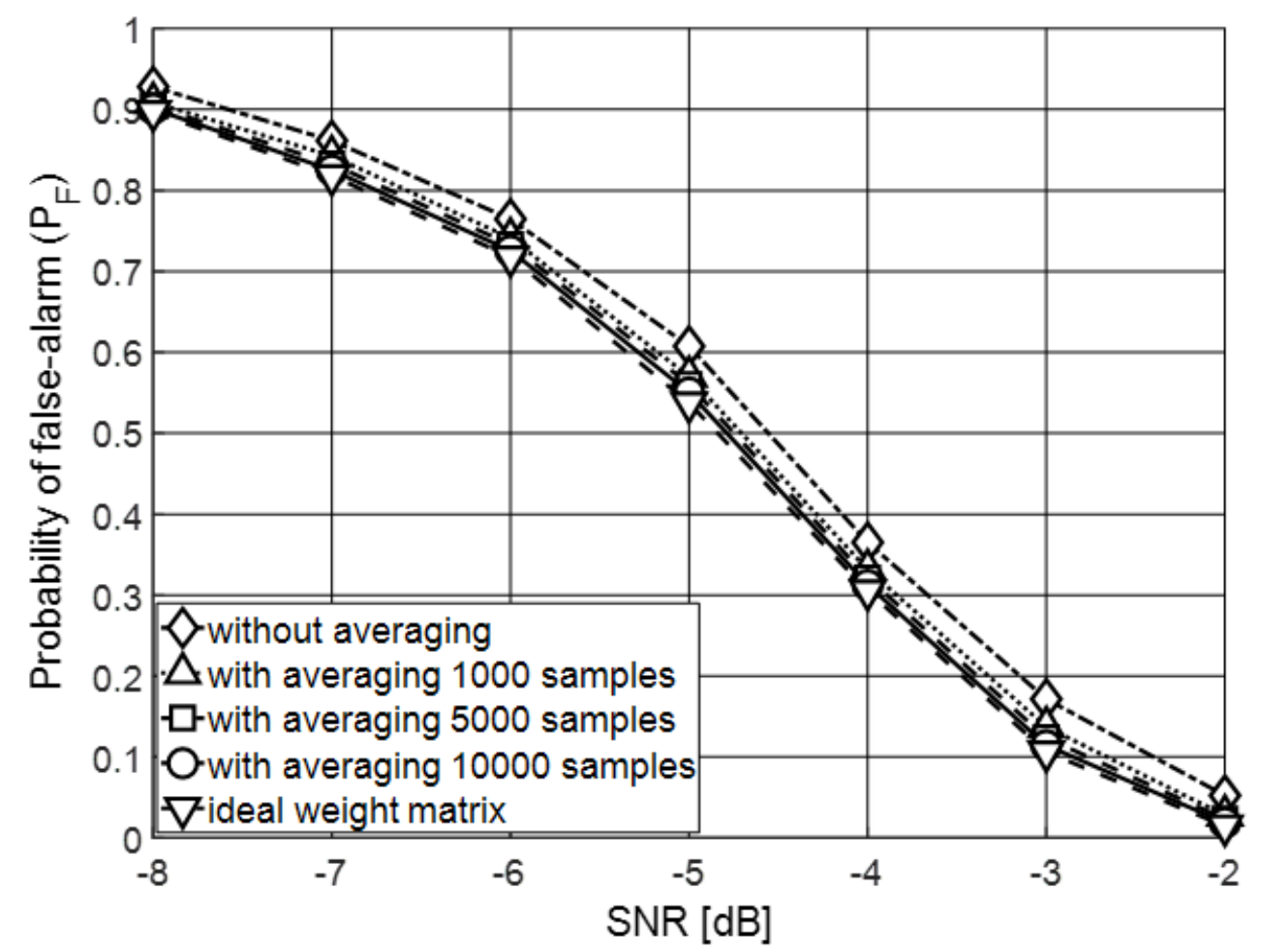

Fig. 5. False-alarm probability of the proposed detector with and without averaging time.

\section{Conclusions}

In this paper, we analyzed how the spatial correlation between antennas affects the sensing performance of the conventional energy detector. Based on that, we proposed the advanced energy detector which can overcome the adverse effect induced by the antenna correlation. Performance between the proposed and conventional detectors is compared, based on an analytic method. Through numerical simulations, it is confirmed that the derived false-alarm and detection probabilities well match the simulation and the sensing performance of the proposed detector is superior to that of the conventional detector in highly correlated antenna environment.

\section{Acknowledgements}

This work was supported by the National Research Foundation of Korea (NRF) grant funded by the Korea government (MSIT) (No. NRF-2021R1A2C2012558).

This work was supported by Road Traffic Authority grant funded by the Korea government (KNPA) (No.POLICE-L-00001-01-101, Development of control system and AI driving capability test for autonomous driving). 


\section{References}

[1] A. Ali and W. Hamouda, "Advances on spectrum sensing for cognitive radio networks: Theory and applications," IEEE Communications Surveys \& Tutorials, vol.19, no.2, pp.1277-1304, Nov. 2016. Article (CrossRef Link)

[2] R. Tandra, and A. Sahai, "SNR walls for signal detection," IEEE Journal of Selected Topics in Signal Processing, vol.2, no.1, pp.4-17, Feb. 2008. Article (CrossRef Link)

[3] S. H. Kamel, M. B. Abd-el-Malek, and S. E. El-Khamy, "Compressive spectrum sensing using chaotic matrices for cognitive radio networks," International Journal of Communication Systems, vol.32, no.6, Dec. 2018. Article (CrossRef Link)

[4] E. S. Hosseini, V. Esmaeelzadeh, R. Berangi, and O. B. Akan, "A correlation-based and spectrum-aware admission control mechanism for multimedia streaming in cognitive radio sensor networks," International Journal of Communication Systems, vol.30, no.3, May. 2015. Article (CrossRef Link)

[5] S. Dikmese, Z. Ilyas, P. C. Sofotasios, M. Renfors, and M. Valkama, "Sparse frequency domain spectrum sensing and sharing based on cyclic prefix autocorrelation," IEEE Journal on Selected Areas in Communications, vol.35, no.1, pp.159-172, Jan. 2017. Article (CrossRef Link)

[6] N. Wang, Y. Gao, F. Yang, Q. Bi, W. Xie, and C. Parini, "Energy detection-based spectrum sensing with constraint region in cognitive LTE systems," Transactions on Emerging Telecommunications Technologies, vol.28, no.11, Mar. 2017. Article (CrossRef Link)

[7] A. Pandharipande and J. P. M. G. Linnartz, "Performance analysis of primary user detection in a multiple antenna cognitive radio," in Proc. of 2007 IEEE International Conference on Communications, Glasgow, UK, 24-28 June 2007. Article (CrossRef Link)

[8] V. Kuppusamy and R. Mahapatra, "Primary user detection in OFDM based MIMO Cognitive Radio," in Proc. of 2008 3rd International Conference on Cognitive Radio Oriented Wireless Networks and Communications (CrownCom 2008), Singapore, 15-17 May 2008.

Article (CrossRef Link)

[9] J. H. Lee, J. H. Baek, and S. H. Hwang, "Collaborative Spectrum Sensing using Energy Detector in Multiple Antenna System," in Proc. of 2008 10th International Conference on Advanced Communication Technology, Gangwon, Korea (South), 17-20 Feb. 2008. Article (CrossRef Link)

[10] D. S. Shiu, G. J. Foschini, M. J. Gans, and J. M. Kahn, "Fading Correlation and Its Effect on the Capacity of Multielement Antenna Systems," IEEE Transaction on Communications, vol.48, no.3, pp.502-513, Mar. 2000. Article (CrossRef Link)

[11] S. Kim, J. Lee, H. Wang, and D. Hong, "Sensing performance of energy detector with correlated multiple antennas," IEEE Signal Processing Letters, vol.16, no.8, pp.671-674, Aug. 2009. Article (CrossRef Link)

[12] Y. C. Liang, Y. Zeng, E. Peh, and A. T. Hoang, "Sensing-throughput tradeoff for cognitive radio networks," IEEE Transactions on Wireless Communications, vol.7, no.4, pp.1326-1337, Apr. 2008. Article (CrossRef Link)

[13] H. Shehata and T. Khattab, "Energy Detection Spectrum Sensing in Full-Duplex Cognitive Radio: The Practical Case of Rician RSI," IEEE Transactions on Communications, vol.67, no.9, pp.6544-6555, May. 2019. Article (CrossRef Link)

[14] H. Wang, G. Noh, D. Kim, S. Kim, and D. Hong, "Advanced sensing techniques of energy detection in cognitive radios," Journal of Communications and Networks, vol.12, no.1, pp.19-29, Feb. 2010. Article (CrossRef Link)

[15] W. Ejaz and M. Ibnkahala, "Multiband spectrum sensing and rewurce allocation for IoT in cognitive 5G networks," IEEE Internet of Things Journal, vol.5, no.1, pp.150-163, Feb. 2018. Article (CrossRef Link)

[16] E. Visotsky, S. Kuffner, and R. Peterson, "On collaborative detection of TV transmissions in support of dynamic spectrum sharing," in Proc. of First IEEE International Symposium on New Frontiers in Dynamic Spectrum Access Networks, 2005 (DySPAN 2005), Baltimore, MD, USA , 8-11 Nov. 2005. Article (CrossRef Link) 
[17] Y. Zeng, C. L. Koh, and Y.-C. Liang, "Maximum eigenvalue detection: theory and application,” in Proc. of 2008 IEEE International Conference on Communications, Beijing, China, pp. 4160-4164, May 2008. Article (CrossRef Link)

[18] R. Zhang, T. J. Lim, Y.-C. Liang, and Y. Zeng, "Multi-antenna based spectrum sensing for cognitive radios: A GLRT approach," IEEE Transactions on Communications, vol. 58, no. 1, pp. 84-88, Jan. 2010. Article (CrossRef Link)

[19] Y. Zeng and Y.-C. Liang, "Eigenvalue-based spectrum sensing algorithms for cognitive radio," IEEE Transactions on Communications, vol. 57, no. 6, pp. 1784-1793, Jun. 2009. Article (CrossRef Link)

[20] C. Liu; H. Li; J. Wang; M. Jin, "Optimal Eigenvalue Weighting Detection for Multi-Antenna Cognitive Radio Networks," IEEE Transactions on Wireless Communications, vol.16, no.4, pp.2083 - 2096, Apr. 2017. Article (CrossRef Link)

[21] S. L. Loyka, "Channel Capacity of MIMO Architecture Using the Exponential Correlation Matrix," IEEE Communications Letters, vol.5, no.9, pp.369-371, Sep. 2001. Article (CrossRef Link)

[22] G. D. Durgin and T. S. Rappaport, "Effects of Multipath Angular Spread on The Spatial Cross-correlation of Received Voltage Envelopes," in Proc. of IEEE 49th Vehicular Technology Conference, Houston, TX, USA, 16-20 May. 1999. Article (CrossRef Link)

[23] S.M. Kay, Fundamentals of Statistical Signal Processing Volume II. Detection Theory, Englewood Cliffs, NJ, USA: PTR Prentice-Hall, 1993.

[24] Z. Quan, S. Chi, and A. H. Sayed, "Optimal Linear Cooperation for Spectrum Sensing in Cognitive Radio Networks," IEEE Journal of Selected Topics in Signal Processing, vol.2, pp.28-40, Feb. 2008. Article (CrossRef Link)

[25] M. S. Alouini, A. Abdi, and M. Kaveh, "Sum of Gamma Variates and performance of Wireless Communication Systems Over Nakagami-Fading Channels," IEEE Transactions on Vehicular Technology, vol.50, pp.1471-1480, Nov. 2001. Article (CrossRef Link)

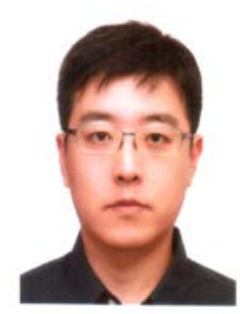

Sungtae Kim received the B.S., M.S., and Ph.D. degrees from the Department of Electronics Engineering at Yonsei University in 2002, 2004, and 2009, respectively. He is currently a researcher with Communication System Examination Division in Korean Intellectual Property Office. His current research interests are 5G/6G and MIMO systems.

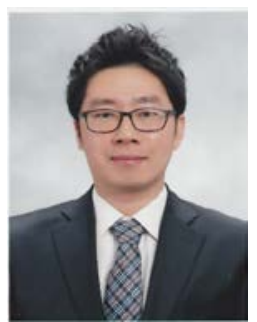

Sungmook Lim received the B.S. and Ph.D. degrees in Electrical and Electronic Engineering at Yonsei University, Seoul, Korea in 2005 and 2012, respectively. From September 2012 to March 2014, he was a Postdoctoral Fellow at Yonsei University where his research interests were in 5G wireless communications. Since March 2014, he joined at Korea National University of Transportation. Currently, he is an Assistant Professor at the Department of electronics Engineering and the Department of IT-Energy Convergence (BK21 FOUR). His current research interests include the field of 5G wireless communications focusing on multicarrier and multi-antenna systems, cooperative relaying, and ITS. 